\title{
Stool-specimen testing practices adopted by clinical microbiology laboratories in the Veneto Region, Italy
}

\author{
Paolo Spolaore', Ugo Fedeli', Ettore De Canale', Giuseppe Bertollo' \\ 'Centro Regionale di Riferimento per il Coordinamento del Sistema Epidemiologico Regionale (CRRC-S.E.R.), Castelfranco \\ Veneto (TV) \\ ${ }^{2}$ Unita Operativa di Microbiologia e Virologia, Azienda Ospedaliera di Padova
}

Key words: Microbiological techniques, Culture media, Infectious diarrheal disease

Indagine conoscitiva sui protocolli analitici utilizzati dai laboratori della Regione Veneto per la diagnostica microbiologica delle gastroenteriti

\section{SUMMARY}

In order to correctly analyze data of laboratory diagnoses of infectious gastroenteritis for epidemiological purposes, a survey on analytical methods applied by hospital-based clinical microbiology laboratories has been conducted in the Veneto Region (Italy).

The survey has been carried out in 2005 through a questionnaire collecting data on laboratory protocols and materials used for faecal specimens analysis.

Laboratories from all the Local Health Units and University Hospitals of the Region returned the questionnaire. Almost all the laboratories routinely tested for the main foodborne pathogens: 23/23 for Salmonella, 22/23 for Shigella and 19/23 for Campylobacter jejuni. A great variety of analytical methods was applied for pathogen isolation; among these is worth of notice the inappropriate use of selenite broth for Shigella enrichment. Among noncultural methods, immunoassays were largely adopted.

The survey allowed to appraise stool-specimen testing practices among laboratories of the Veneto Region; overall the compliance with guidelines proposed by the main national and international scientific societies resulted rather good.

\section{INTRODUZIONE}

Il Centro Regionale di Riferimento per il Coordinamento del Sistema Epidemiologico Regionale (C.R.R.C.-S.E.R.) ha avviato nel 2003 la raccolta sistematica dei dati contenuti negli archivi microbiologici dei laboratori ospedalieri e la loro integrazione con i dati provenienti dalle SDO (Schede di Dimissione Ospedaliera), al fine di promuovere programmi di controllo e prevenzione delle infezioni che complicano l'assistenza ospedaliera.

La costituzione di un archivio microbiologico regionale offre enormi potenzialità per la sorveglianza epidemiologica anche alle infezioni comunitarie; tra queste un ambito di notevole interesse è quello delle infezioni di origine alimentare. Il CRRC-SER infatti, nell'ambito del Piano Regionale Veneto per la Sicurezza Alimentare, ha avviato un progetto che mira ad integrare le fonti informative esistenti (notifiche obbligatorie, SDO, archivi microbiologici) così da tracciare un quadro epidemiologico complessivo delle infezioni a trasmissione alimentare.

Alcuni problemi rendono però difficile una corretta elaborazione dei dati, in particolare quelli derivanti dalla eterogeneità dei protocolli analitici utilizzati dai laboratori di microbiologia. Altro elemento di criticità è legato al fatto che, diversamente dalle ricerche effettuate "di routine" sulla base di protocolli analitici prestabiliti, le "ricerche specifiche" esigono procedure diverse per la "esportazione" dei risultati e la successiva integrazione nell'archivio microbiologico regionale.

Al fine di interpretare ed elaborare correttamente i dati provenienti dagli archivi microbiologici dei laboratori che partecipano al progetto di sorveglianza epidemiologica delle infezioni, il C.R.R.C.-S.E.R. ha condotto un'indagine conoscitiva sui protocolli adottati dai laboratori di microbiologia di tutte le Aziende ULSS ed Ospedaliere della Regione Veneto, per la diagnostica microbiologica delle gastroenteriti. 


\section{MATERIALI E METODI}

L'indagine è stata condotta, nel 2005, presso i Laboratori (Servizi/Settori di Microbiologia) di tutte le Aziende ULSS e Ospedaliere della Regione Veneto, mediante somministrazione di una specifica scheda informativa sulla ricerca degli enteropatogeni nei campioni fecali.

La lista degli enteropatogeni comprendeva: Salmonella spp., Shigella spp., Campylobacter jejuni, Yersinia enterocolitica, E coli O157:H7, Rotavirus, Norovirus, Giardia lamblia, Cryptosporidium spp., ed una categoria residuale chiamata "Altri".

Non sono state raccolte informazioni in merito all'idoneità dei campioni ad essere processati secondo i protocolli analitici indicati dai laboratori. Ciò ritenendo implicita l'osservanza, da parte del microbiologo, delle buone pratiche di laboratorio tra cui la valutazione pre-analitica del campione per verificarne la corretta conservazione e l'idoneità ad essere processato secondo il protocollo prestabilito.

L'analisi è stata condotta mediante aggregazione di dati omogenei su matrici predefinite (tabella 1 e tabella 2) ed orientate all'ottenimento di informazioni rispondenti agli obiettivi dell'indagine.

\section{RISULTATI}

Hanno risposto all'indagine i Laboratori (23 Servizi/Settori di Microbiologia) di tutte le Aziende ULSS ed Ospedaliere (Padova e Verona) della Regione Veneto.

I risultati dell'indagine sono riassunti in tabella 1 per quanto concerne lo "spettro" degli enteropatogeni ricercati ed in tabella 2 per quanto si riferisce ai metodi analitici utilizzati.

Come si può osservare in tabella 1 , i risultati dell'indagine dimostrano che la quasi totalità dei laboratori di microbiologia effettuano la ricerca $d i$ routine dei tre principali enteropatogeni: 23/23 ricercano Salmonella spp., 22/23 Shigella spp. e 19/23 Campylobacter jejuni.

Solo una minoranza di laboratori dichiara di effettuare la ricerca routinaria di Yersinia enterocoliti- ca (3/23), E. coli $\mathrm{O} 157: \mathrm{H} 7$ (1/23), Rotavirus in piccoli pazienti di età inferiore ai due anni $(1 / 23)$, Giardia lamblia (4/23) e Cryptosporidium spp. $(1 / 23)$.

La maggior parte dei laboratori effettua la ricerca di alcuni enteropatogeni, esclusivamente su specifica richiesta del Clinico: Yersinia enterocolitica (20/23), E. coli O157:H7 (17/23), Rotavirus (22/23), Giardia lamblia (18/23) e Cryptosporidium spp. (19/23).

Un solo laboratorio esige una specifica richiesta del Clinico per effettuare la ricerca di Shigella spp., mentre, 4/23 la richiedono per effettuare la ricerca di Campylobacter jejuni.

Nessun laboratorio ricerca di routine Norovirus nei campioni fecali e solo 4/23 li ricercano su specifica richiesta del Clinico.

Solo alcuni laboratori dichiarano di non effettuare "in nessun caso" la ricerca di particolari enteropatogeni: $3 / 23$ E. coli O157:H7, 11/23 Norovirus e 2/23 Cryptosporidium spp. Riguardo ai materiali ed ai metodi utilizzati per la ricerca degli enteropatogeni, come si può osservare in tabella 2 , tutti i laboratori utilizzano metodi colturali per effettuare la ricerca di Salmonella spp., Shigella spp., Campylobacter jejuni, Yersinia enterocolitica ed E. coli $\mathrm{O} 157: \mathrm{H7}$

In tutti i laboratori la ricerca di Salmonella spp. è preceduta da un "arricchimento" del campione prima dell'isolamento primario: 17/23 laboratori utilizzano il brodo Selenite, $1 / 23$ il brodo Tetrationato, 1/23 brodo Rappaport e 1/23 il brodo Gram negativi.

Per l'arricchimento di Shigella spp., 10/23 laboratori utilizzano impropriamente il brodo Selenite che contiene inibitori per alcune specie di Shigella, 1/23 laboratori utilizza il brodo Tetrationato, 1/23 utilizza il brodo Rappaport ed $1 / 23$ il brodo Gram negativi, unico quest'ultimo che consente la crescita sia di Salmonella spp. sia di Shigella spp.

Campylobacter jejuni è isolato da 10/23 laboratori mediante Agar Campylobacter selettivo; 4/23 lo isolano mediante Agar Karmali ed un solo labora-

Tabella I. Criteri adottati dai Servizi/Settori di Microbiologia della Regione Veneto per la ricerca dei microrganismi enteropatogeni nei campioni fecali

\begin{tabular}{lcccc}
\hline Enteropatogeno & di routine* & su specifica richiesta* & in nessun caso* & non specificato * \\
\hline Salmonella spp. & $23(100 \%)$ & 0 & 0 & 0 \\
\hline Shigella spp. & $22(95.6 \%)$ & 1 & 0 & 0 \\
\hline Campylobacter jejuni & $19(82.6 \%)$ & 4 & 0 & 0 \\
\hline Yersinia enterocolitica & $3(13 \%)$ & $20(86.9 \%)$ & 0 & 0 \\
\hline E. coli OI57:H7 & $\mathrm{I}$ & $17(73.9 \%)$ & 3 & 2 \\
\hline Rotavirus & $\mathrm{I}$ & $22(95.6 \%)$ & 0 & 0 \\
\hline Norovirus & 0 & 4 & 0 & $8(34.8 \%)$ \\
\hline Giardia lamblia & 4 & $18(78.3 \%)$ & 2 & 1 \\
\hline Cryptosporidium spp. & $\mathrm{I}$ & $19(82.6 \%)$ & 0 & 1 \\
\hline Altri & 0 & $10(43.5 \%)$ & & 13 \\
\hline
\end{tabular}

* $N^{\circ}$ Laboratori che adottano il criterio (\%). 
torio utilizza l'Agar Rappaport.

Nonostante l'arricchimento del campione non sia raccomandato per la ricerca routinaria di Campylobacter jejuni, 3/23 laboratori lo effettuano: 1 mediante brodo Tioglicolato, 1 mediante brodo cuore cervello ed 1 mediante brodo non specificato.

L'isolamento di Yersinia enterocolitica è effettuato mediante Agar CIN dalla maggioranza dei laboratori (15/23); 2/23 laboratori dichiarano di utilizzare Agar MacConkey; 1/23 Agar CIN e Agar MacConkey mentre $1 / 23$ utilizza Agar MacConkey e Agar Salmonella Shigella.

L'arricchimento di Yersinia enterocolitica non è raccomandato "di routine" ed infatti solo 1/23 laboratori dichiara di adottare questa procedura prima dell'inoculo sui terreni di isolamento primario.

Su 18 laboratori che dichiarano di effettuare la ricerca di E. coli $\mathrm{O} 157: \mathrm{H} 7$, prevalentemente su richiesta specifica, 14 utilizzano Agar MacConkey Sorbitolo, 2 Agar MacConkey, 1 Agar Hektoen Enteric ed 1 "non specificato".

Come si può osservare in tabella 2, alcuni entero- patogeni, fra quelli censiti, vengono ricercati con metodi non colturali: Rotavirus, Norovirus, Giardia lamblia e Cryptosporidium spp..

La maggioranza dei laboratori (12/23) effettuano la ricerca di Rotavirus mediante immunocromatografia; 4/23 laboratori utilizzano test immunoenzimatici e $2 / 23$ test di agglutinazione al Latice. Solo 4/23 laboratori hanno dichiarato di poter garantire la ricerca di Norovirus nei campioni fecali, utilizzando metodi immunoenzimatici (2/4) o metodi immunocromatografici (1/4).

Giardia lamblia viene ricercata dai laboratori prevalentemente (13/22) mediante osservazione microscopica a fresco previo arricchimento del campione; 2/22 laboratori utilizzano l'immunofluorescenza indiretta; 2/22 l'immunocromatografia e solo 1/22 l'immunoenzimatica.

Per la ricerca di Cryptosporidium, infine, 9/20 laboratori effettuano la ricerca microscopica sui campioni fecali previa colorazione con il metodo di Ziehl Neelsen modificato; 2/20 laboratori utilizzano l'immunofluorescenza indiretta; $2 / 20$ l'immunocromatografia ed 1/20 l'immunoenzimatica.

Tabella 2. Metodi utilizzati dai Servizi/Settori di Microbiologia della Regione Veneto per la ricerca dei microrganismi enteropatogeni nei campioni fecali.

\begin{tabular}{|c|c|c|c|}
\hline Enteropatogeno & Colturale* & Arricchimento* & Altri metodi * \\
\hline Salmonella spp. & $\begin{array}{c}\text { 5/23 MAC+SSA, 4/23 XLD, } \\
\text { 4/23 SSA, 3/23 HEA, } 2 / 23 \\
\text { HEA+SSA, I/23 XLD+SSA, } \\
\text { I/23 SCM, } 3 / 23 \text { n.s }\end{array}$ & $\begin{array}{c}\text { I7/23 BS, I/23 BT, } \\
\text { I/23 BR, I/23 GNB, } \\
\text { 3/23 n.s. }\end{array}$ & \\
\hline Shigella spp. & $\begin{array}{c}\text { 5/23 MAC+SSA, } 4 / 23 \text { XLD, } \\
\text { 5/23 SSA, } 2 / 23 \text { HEA+SSA, } \\
\text { I/23 XLD+SSA, I/23 } \\
\text { MAC+XLD, I/23 HEA, I/23 } \\
\text { SCM, } 3 / 23 \text { n.s. }\end{array}$ & $\begin{array}{l}\text { I0/23 BS, I/23 BT, } \\
\text { I/23 BR, I/23 GNB, } \\
\text { I0/23 n.s. }\end{array}$ & \\
\hline Campylobacter jejuni & $\begin{array}{c}\text { I0/23 CAMPY, 4/23 KA, I/23 } \\
\text { AP, I/23 n.s }\end{array}$ & $\begin{array}{l}\text { I/23 FTM, I/23 BHIA, } \\
\text { I/23 n.s, } 20 / 23 \text { n.e. }\end{array}$ & \\
\hline Yersinia enterocolitica & $\begin{array}{c}\text { I5/23 CIN, } 2 / 23 \text { MAC, I/23 } \\
\text { CIN+MAC, I/23 MAC+SSA, } \\
\text { 4/23 n.s. }\end{array}$ & I/23 PW, 22/23 n.e. & \\
\hline E. coli OI57:H7 & $\begin{array}{c}\text { I4/I8 MACs, 2/18 MAC, I/I8 } \\
\text { HEA, I/I8 n.s. }\end{array}$ & 18/18 n.e. & \\
\hline Rotavirus & & & $\begin{array}{l}\text { I2/23 IC, 4/23 EIA, } \\
\text { 2/23 LA, 5/23 n.s. }\end{array}$ \\
\hline Norovirus & & & $\begin{array}{c}\text { 2/4 EIA, I/4 IC, I/4 } \\
\text { n.s. }\end{array}$ \\
\hline Giardia lamblia & & & $\begin{array}{c}\text { I3/22 micro a fresco, } \\
2 / 22 \text { IFA, } 2 / 22 \text { IC, I/22 } \\
\text { EIA }\end{array}$ \\
\hline Cryptosporidium spp & & & $\begin{array}{c}9 / 20 \mathrm{ZN} \bmod , 2 / 20 \\
\text { IFA, } 2 / 20 \text { IC, I/20 } \\
\text { EIA, } 6 / 20 \text { n.s. }\end{array}$ \\
\hline
\end{tabular}

* $N^{\circ}$ Laboratori che utilizzano il metodo / $N^{\circ}$ laboratori che hanno risposto all'indagine. Abbreviazioni terreni di coltura: MAC:Agar MacConkey; SSA:Agar Salmonella Shigella; XLD:Agar Xilosio Lattosio Desossicolato; HEA:Agar Hektoen Enteric; SCM: Agar cromogenico; CAMPY: Agar Campylobacter; KA: Agar Karmali; AP: Agar Preston; CIN: Agar Cefsulodin-lgarsanNovobiocin; MACs: Agar MacConkey Sorbitolo; BS: Brodo Selenite; BT: Brodo Tetrationato; BR: Brodo Rappaport; GNB: Brodo Gram Negativi; FTM: Brodo Tioglicolato; BHIA: Brodo Cuore Cervello; PW:Acqua Peptonata; n.s. non specificato; n.e. non effettuato

Abbreviazioni metodi analitici non colturali: IC: Immunocromatografia; EIA: Immunoenzimatica; LA: Agglutinazione al Latice; IFA: Immunofluorescenza; Z.N. mod.: Ziehl-Neelsen modificato. 


\section{DISCUSSIONE}

Questi dati dimostrano che la maggioranza dei laboratori della Regione Veneto assicura la diagnostica microbiologica delle gastroenteriti applicando procedure e linee guida raccomandate dalla comunità scientifica $(1,2,3)$.

Come raccomandato infatti, la quasi totalità dei Laboratori effettua la ricerca routinaria dei 3 principali enteropatogeni: Salmonella spp., Shigella spp. e Campylobacter jejuni. Solo 4 laboratori che effettuano la ricerca di Campylobacter jejuni esclusivamente "su richiesta specifica" dovranno adeguarsi ai protocolli analitici ormai consolidati ed effettuare la ricerca routinaria di questo importante enteropatogeno.

Per quanto riguarda gli altri enteropatogeni censiti con la scheda informativa, quasi tutti i laboratori sono in grado di assicurarne la ricerca, con la sola eccezione di Norovirus.

Naturalmente la ricerca di Norovirus è molto specialistica e tecnicamente complessa, tuttavia, negli ultimi anni, sono andati sviluppandosi alcuni interessanti sistemi analitici immunoenzimatici che ancora non sostituiscono il sistema di riferimento rappresentato dalla immunoelettromicroscopia ma che potrebbero già essere utilizzati per lo screening dei campioni in corso di epidemia. È importante registrare, inoltre, che la maggior parte dei laboratori è in grado di assicurare la ricerca di E. coli O157:H7, fino a pochi anni fa appannaggio di pochissimi centri di ricerca che lavoravano su progetti finalizzati. L'importanza di questo sierotipo batterico non riguarda tanto la sua incidenza nella casistica complessiva delle gastroenteriti ma, in particolare, la gravità delle complicanze possibili: la Colite Emorragica e la Sindrome Emolitico Uremica $(4,5)$.

In merito ai sistemi colturali utilizzati dai vari laboratori, si registra una notevole difformità nella scelta dei terreni colturali di isolamento primario anche se complessivamente rispondenti alle raccomandazioni ed alle linee guida delle Società Scientifiche. Sarebbe tuttavia auspicabile una maggiore standardizzazione nell'uso dei terreni solidi selettivi di primo isolamento possibilmente orientata all'uso di due terreni: uno a bassa l'altro a media selettività (1).

I risultati purtroppo mostrano l'uso prevalente del brodo Selenite per l'arricchimento selettivo di Shigella spp. nei campioni fecali, nonostante questo brodo contenga inibitori per alcune specie di Shigella.

Fra i metodi "non colturali" utilizzati per la ricerca degli enteropatogeni si conferma la notevole diffusione dei test immunocromatografici che sono molto rapidi e sono in continuo migliora- mento delle loro performance di sensibilità e specificità.

In conclusione, l'indagine ha consentito di approfondire le conoscenze sul processo analitico dei laboratori della Regione Veneto riguardo alla diagnostica microbiologica delle gastroenteriti, ha evidenziato la sostanziale aderenza alle linee guida nazionali ed internazionali ed ha conferito al C.R.R.C.-S.E.R. informazioni e garanzie per una corretta elaborazione dei dati a fini epidemiologici.

\section{BIBLIOGRAFIA}

1. Farmer III JJ, Kelly MT. In: Manual of Clinical Microbiology, Balows A, Hansler WJ, Herrmann KL, Isenberg HD, Shadomy HJ Eds, Fifth Edition. American Society for Microbiology, Washington DC 1991; 360-83.

2. Health Protection Agenzy. Investigation of faeces specimens for bacteria pathogens. National Standard Method, 2004, BSOP 30 Issue5.

3. Isemberg HD. In: Clinical Microbiology Procedures Handbook, Isemberg HD Eds. American Society for Microbiology, Washington DC 1992; 1.10.1 1.10.12.

4. Neill MA, Agosti J, Rosen H. Haemorrhagic colitis with Escherichia coli O157:H7 preceding adult haemolytic uremic syndrome. Arch Intern Med 1985; 145: 2215-7.

5. Tserenpuntsag B, Chang HG, Smith PF, Morse DL. Hemolytic uremic syndrome risk and Escherichia coli O157:H7. Emerg Infect Dis 2005; 11: 1955-7.

\section{RINGRAZIAMENTI}

Si ringraziano i Direttori delle Unità di Microbiologia e Chimica Clinica della Regione Veneto ed i loro Collaboratori che hanno aderito a questa indagine finalizzata ad una corretta lettura degli archivi microbiologici. 